\title{
Influência de diferentes substratos e umidade sobre o crescimento e o número de ovos produzidos por Subulina octona (Brugüière) (Mollusca, Subulinidae), sob condições de laboratório
}

\author{
Sthefane D'ávila ${ }^{1} \&$ Elisabeth C. de A. Bessa ${ }^{2}$ \\ 1 E-mail: sthefanedavila@hotmail.com \\ 2 Departamento de Zoologia, Instituto de Ciências Biológicas, Universidade Federal de Juiz de Fora. Campus Universitário, \\ 36036330 Juiz de Fora, Minas Gerais, Brasil.
}

\begin{abstract}
Influence of moisture on growth and egg production by Subulina octona (Brugüière) (Mollusca, Subulinidae), reared in different substrates, under laboratorial conditions. The aim of this study was to observe the influence of substrate moisture on growth, number of eggs produced and shell length of Subulina octona (Brugüiière, 1789)in its first reproductive event. For this, two groups of moluscs were observed under laboratorial conditions. The trated group, manteined in substrate moinstened at five-days intervals and the control group, manteined in substrate moistened at one-day intervals, were both constituted by 210 newly hatched individuals distributed in boxes with different substrates. The box one and control one contained sand, the box two and control two, clay and the box three and control three, humus. Each box contained 35 snails. The individuals of the control group exhibited a faster growth rhythm than those of the trated group. The substrate moisture was a major influence over the individuals reared in sand and clay. It's probable due to the fact that these substrates loose water by evaporation more quickly than humus. The land snails loose water by their skin and by the same means they rehidrate by contact. So, the humidity of the substrate on wich the snails live influence their homeostasis. Substrates that retain water for a smaler time period offer fewer oportunities to rehidration. The waterloos by the skin, without later rehidration can causes changes in hemolinph osmolarity, with implications over the feedind control, hearth beating and locomotion. By this way, snails submited to dessication exhibes reduced activity and consequently smaler growth and produtivity.
\end{abstract}

KEY WORDS. humidity, land snail, reproduction.

RESUMO. O objetivo deste estudo foi verificar a influência da umidade do substrato sobre o crescimento e a reprodução de Subulina octona (Brugüière, 1789), criada em areia, argila e terra vegetal. Sobre o grupo experimental, constituído por três grupos tratados (substrato umedecido com $10 \mathrm{ml}$ de água a intervalos de cinco dias) e três grupos controle (substrato umedecido com $10 \mathrm{ml}$ de água a intervalos de um dia), foram realizadas observações a cerca do crescimento, expresso pelo comprimento da concha; do número de ovos produzidos e do comprimento da concha no primeiro evento reprodutivo. $O$ grupo tratamento e o grupo controle constituíram-se de 210 indivíduos recém-eclodidos, distribuídos aleatoriamente em caixas diferenciais em função do substrato utilizado: caixa 1 e controle 1, com areia; caixa 2 e controle 2, com argila; caixa 3 e controle 3, com terra vegetal. Cada caixa continha 35 indivíduos. Os indivíduos do grupo controle (substrato umedecido a intervalos de um dia) apresentaram um ritmo de crescimento mais rápido. A umidade do substrato foi um fator de maior influência sobre a reprodução dos indivíduos criados em caixas com areia e argila. Houve diferença significativa entre as médias do comprimento da concha e do número de ovos produzidos, no primeiro evento reprodutivo dos indivíduos da caixa 1 (areia) e do grupo controle 1. Os indivíduos da caixa 2 (argila) apresentaram menor comprimento de concha no primeiro evento reprodutivo do que os indivíduos do grupo controle 2. Os gastrópodes terrestres perdem água pelo tegumento e, do mesmo modo, se reidratam pelo tegumento, através da chamada reidratação por contato. Neste contexto, a umidade do substrato influencia a manutenção da homeostase por estes organismos e conseqüentemente seu crescimento e reprodução.

PALAVRAS CHAVE. Moluscos terrestres.

Uma característica ecológica conspícua dos moluscos pulmonados é a grande dependência da umidade, que se reflete no comportamento, período de atividade, preferência por habitat, atividade reprodutiva e parece ter sido uma das principais pres- 
sões seletivas atuando sobre esse grupo (Elwell \& Ulmer 1971, Dimitrieva 1975, Pieri \& Jurberg 1981). Este fato é evidenciado pelas diversas adaptações comportamentais e fisiológicas exibidas por estes animais, as quais representam soluções eficientes para os problemas relacionados às condições ambientais.

De uma forma geral, os gastrópodes pulmonados apresentam um grande poder de resistência e adaptação a períodos de condições adversas (Pieri \& Jurberg 1981). O comportamento agregativo (DundeE et al. 1975); a seleção de substratos com níveis adequados de umidade para a oviposição, o enterramento desses ovos em cavidades no solo (LEAHY 1983); a formação de espermatóforos (WIKTOR 1987); a deposição de fezes que apresentam constituição distinta das fezes usualmente formadas sobre os ovos (RAUT \& PANIGRAHI 1988); a estivação e retração da massa cefalopodal no interior da concha (Elwell \& UlmER 1971, RichARDOT 1977a, b, 1978) são algumas das estratégias que permitem a sobrevivência e o sucesso reprodutivo desses organismos, sob condições desfavoráveis de umidade.

A umidade influencia aspectos do ciclo de vida dos moluscos pulmonados, tais como a alimentação, o ritmo de batimento cardíaco, a locomoção, o crescimento, a produtividade, a espermatogênese, a produção e incubação dos ovos (HodAsI 1979, 1982, LeAHy 1980, TuAN \& SimÕes 1984, CoOK 2001, FURTADO et al. 2002).

O objetivo do presente estudo foi verificar a influência da umidade do substrato sobre o crescimento e a produção de ovos por Subulina octona, mantida em diferentes substratos.

\section{MATERIAL E MÉTODOS}

Este estudo foi realizado durante o período de 22 de novembro de 2001 a 25 de janeiro de 2002. A metodologia utilizada para a obtenção e manutenção dos moluscos, assim como a formação dos grupos experimentais e os tratamentos empregados foi idêntica à descrita por D'ÁvILA \& BESSA (2005a), em um estudo sobre a influência do substrato sobre a reprodução de $S$. octona.

O grupo experimental foi constituído por três grupos tratados (substrato umedecido com $10 \mathrm{ml}$ de água de torneira a intervalos de cinco dias) e três grupos controle (substrato umedecido com $10 \mathrm{ml}$ de água de torneira a intervalos de um dia). Foram realizadas observações a cerca do crescimento, expresso pelo comprimento da concha; do número de ovos produzidos e do comprimento da concha no primeiro evento reprodutivo. O grupo tratamento e o grupo controle constituíram-se de 210 indivíduos recém-eclodidos, distribuídos aleatoriamente em caixas diferenciais em função do substrato utilizado: caixa 1 e controle 1, com substrato arenoso; caixa 2 e controle 2 , com substrato argiloso; caixa 3 e controle 3 , com terra vegetal. Cada caixa continha 35 indivíduos. O comprimento da concha dos moluscos dos grupos tratados e do grupo controle foi medido quinzenalmente, com o auxílio de um paquímetro, até o $30^{\circ}$ dia de vida. Ao primeiro aparecimento de ovos, visíveis no útero por transparência da concha, foram registrados o compri- mento da concha e o número de ovos produzidos por cada indivíduo, de cada grupo. Os dados foram analisados comparativamente. Para a análise estatística dos dados foi utilizado o teste de análise de variância (One way ANOVA), seguido pelo teste de Tamhane, com intervalo de confiança de 95\%.

\section{RESULTADOS E DISCUSSÃO}

Durante o período de realização do experimento a temperatura máxima variou de $21-31^{\circ} \mathrm{C}$, a temperatura mínima de $20-28^{\circ} \mathrm{C}$ e a umidade relativa do ar de $47-92 \%$.

São apresentadas na tabela I as medidas do comprimento da concha dos indivíduos dos grupos tratados e respectivos grupos controle, no início do experimento (recém-eclodidos), aos 15 dias de vida e aos 30 dias de vida.

Os indivíduos do grupo controle (substrato umedecido a intervalos de um dia) apresentaram um ritmo de crescimento mais rápido que aqueles dos grupos tratados (substrato umedecido a intervalos de cinco dias). O teste ANOVA $(p<0,05)$ demonstrou haver diferença significativa entre as médias do comprimento da concha dos indivíduos do grupo tratado: caixas 1 (areia) (F: 79,995; sig.: 0,000), 2 (argila) (F: 161,626; sig.: 0,000) e 3 (terra vegetal) (F: 18,811; sig.: 0,000) e seus respectivos grupos controle, aos 30 dias de vida.

Quando submetidos a condições desfavoráveis de umidade, os moluscos terrestres exibem uma série de mudanças comportamentais, fisiológicas e bioquímicas, que caracterizam o estado de estivação (Richardot 1977a, b, Storey 2002). Essas mudanças têm início com a seleção de locais protegidos, seguida pela retração da massa cefalopodal no interior da concha e imobilização, que persiste até o retorno das condições favoráveis (ElWELl \& Ulmer 1971, Richardot 1977a, b, 1978). Durante a estivação, os moluscos não se alimentam e as suas reservas de energia são utilizadas apenas para sustentar o metabolismo basal. Conseqüentemente, não há energia disponível para o crescimento (STOREY 2002).

Nas condições do presente estudo, a diferença na umidade do substrato entre a caixa 3 (terra vegetal) e o grupo controle 3 , não determinou diferenças no número de ovos produzidos e no comprimento da concha no primeiro evento reprodutivo dos indivíduos dos respectivos grupos. Não houve diferença significativa entre as médias de comprimento da concha e número de ovos produzidos no primeiro evento dos indivíduos da caixa 3 (terra vegetal) e do grupo controle 3 (Tabs II e III).

A umidade do substrato foi um fator de maior influência sobre a reprodução dos indivíduos criados em caixas com areia e argila. Houve diferença significativa entre as médias do comprimento da concha (F: 40,337; sig.: 0,000) e do número de ovos produzidos (F: 4,166; sig.: 0,046), no primeiro evento reprodutivo dos indivíduos da caixa 1 (areia) e do grupo controle 1. Os indivíduos da caixa 2 (argila) apresentaram menor comprimento de concha no primeiro evento reprodutivo do que os indivíduos do grupo controle 2 (F: 34,734; sig.: 0,000). É provável que estes dois substratos percam água por evapora- 
Tabela I. Comprimento da concha (em milímetros) de indivíduos da espécie Subulina octona, recém-eclodidos, aos 15 e 30 dias de vida, mantidos em diferentes substratos, umedecidos a intervalos de um dia (grupos controle) e a intervalos de cinco dias (grupos tratados).

\begin{tabular}{|c|c|c|c|c|c|}
\hline & Mínimo & Máximo & Média & Desvio padrão & Coeficiente de variação (\%) \\
\hline \multicolumn{6}{|c|}{ Comprimento da concha $-1^{\circ}$ dia de vida } \\
\hline Caixa 1 (areia) & 2,0 & 3,0 & 2,78 & 0,31 & 11,24 \\
\hline Controle 1 (areia) & 1,0 & 3,0 & 2,12 & 0,40 & 19,18 \\
\hline Caixa 2 (argila) & 2,0 & 3,0 & 2,81 & 0,35 & 12,75 \\
\hline Controle 2 (argila) & 1,5 & 3,0 & 2,41 & 0,50 & 21,00 \\
\hline Caixa 3 (terra vegetal) & 2,0 & 3,0 & 2,65 & 0,45 & 17,27 \\
\hline Controle 3 (terra vegetal) & 1,0 & 3,0 & 2,07 & 0,62 & 29,93 \\
\hline \multicolumn{6}{|c|}{ Comprimento da concha $-15^{\circ}$ dia de vida } \\
\hline Caixa 1 (areia) & 5,0 & 7,0 & 6,42 & 0,62 & 9,77 \\
\hline Controle 1 (areia) & 4,0 & 8,5 & 6,47 & 1,11 & 17,21 \\
\hline Caixa 2 (argila) & 4,0 & 8,0 & 6,33 & 0,73 & 11,60 \\
\hline Controle 2 (argila) & 5,0 & 8,0 & 6,60 & 0,83 & 12,70 \\
\hline Caixa 3 (terra vegetal) & 2,0 & 6,5 & 4,68 & 1,54 & 32,96 \\
\hline Controle 3 (terra vegetal) & 2,0 & 6,0 & 3,46 & 1,19 & 34,35 \\
\hline \multicolumn{6}{|c|}{ Comprimento da concha $-30^{\circ}$ dia de vida } \\
\hline Caixa 1 (areia) & 5,5 & 9,0 & 7,96 & 1,00 & 12,66 \\
\hline Controle 1 (areia) & 8,0 & 13,0 & 10,66 & 0,95 & 8,96 \\
\hline Caixa 2 (argila) & 5,5 & 9,5 & 7,93 & 0,86 & 10,94 \\
\hline Controle 2 (argila) & 9,0 & 12,5 & 10,81 & 1,26 & 11,70 \\
\hline Caixa 3 (terra vegetal) & 3,0 & 8,0 & 5,93 & 1,65 & 27,90 \\
\hline Controle 3 (terra vegetal) & 4,0 & 11,0 & 7,89 & 1,58 & 20,13 \\
\hline
\end{tabular}

Tabela II. Comprimento da concha (em milímetros), no primeiro evento reprodutivo de indivíduos da espécie Subulina octona, mantidos em diferentes substratos, umedecidos a intervalos de um dia (grupos controle) e a intervalos de cinco dias (grupos tratados).

\begin{tabular}{lccccc}
\hline \multicolumn{1}{c}{ Comprimento da concha } & Mínimo & Máximo & Média & Desvio padrão & Coeficiente de variação (\%) \\
\hline Caixa 1 (areia) & 8 & 15,0 & 12,15 & 1,40 & 11,54 \\
Controle 1 (areia) & 13 & 16,0 & 14,08 & 0,90 & 6,38 \\
Caixa 2 (argila) & 9 & 13,5 & 12,11 & 1,10 & 9,11 \\
Controle 2 (argila) & 11 & 17,0 & 14,12 & 1,42 & 10,09 \\
Caixa 3 (terra vegetal) & 7 & 13,0 & 11,02 & 1,82 & 16,57 \\
Controle 3 (terra vegetal) & 8 & 14,0 & 11,46 & 1,37 & 12,03 \\
\hline
\end{tabular}

ção mais rapidamente do que a terra vegetal e por isso, o fornecimento de água em intervalos de cinco dias determinou uma condição desfavorável de umidade, afetando a reprodução dos moluscos.

Os gastrópodes terrestres perdem água pelo tegumento e, do mesmo modo, se reidratam pelo tegumento, através da chamada reidratação por contato (Cook 2001). Neste contexto, a capacidade de retenção de água do substrato sobre o qual os moluscos vivem influencia a manutenção da homeostase por estes organismos. Substratos que retêm água por menos tempo fornecem menor oportunidade de reidratação por contato, durante períodos desfavoráveis. A perda de água pelo tegumento, sem reidratação posterior, pode levar a uma mudança na osmolaridade da hemolinfa, com implicações sobre o controle da alimentação, do batimento cardíaco e da locomoção (Cook 2001). Dessa forma, moluscos submetidos à dessecação podem ter sua atividade reduzida e conseqüentemente um menor crescimento e produtividade (HodAsi 1979, 1982). LEAHY (1980) observou que com a diminuição da umidade, Bradybaena similaris Férussac, 1821 (Xanthonychidae) diminui progressivamente sua atividade, até tornar-se imóvel no substrato. TuAN \& SiMÕES (1989) verificaram que sob condições de dessecação, o processo meiótico durante a espermatogênese de Biomphalaria tenagophila Orbigny, 1835 (Planorbiidae) é len- 
Tabela III. Número de ovos produzidos, no primeiro evento reprodutivo de indivíduos da espécie Subulina octona, mantidos em diferentes substratos, umedecidos a intervalos de um dia (grupos controle) e a intervalos de cinco dias (grupos tratados).

\begin{tabular}{lccccc}
\hline \multicolumn{1}{c}{ Número de ovos } & Mínimo & Máximo & Média & Desvio padrão & Coeficiente de variação (\%) \\
\hline Caixa 1 (areia) & 1 & 3 & 2,03 & 0,34 & 16,88 \\
Controle 1 (areia) & 1 & 4 & 2,55 & 1,05 & 41,03 \\
Caixa 2 (argila) & 1 & 3 & 2,04 & 0,35 & 14,01 \\
Controle 2 (argila) & 1 & 6 & 2,28 & 1,05 & 46,22 \\
Caixa 3 (terra vegetal) & 1 & 4 & 1,92 & 0,73 & 37,85 \\
Controle 3 (terra vegetal) & 1 & 4 & 2,10 & 0,91 & 43,49 \\
\hline
\end{tabular}

to. FURTADO et al. (2002) observaram aparecimento de ovos mais tardio em B. similaris, em função de uma baixa umidade relativa do ar, durante seus experimentos. Raut \& GHose (1980) analisaram a influência de fatores abióticos sobre o tempo de incubação dos ovos de Achatina fulica Bowdich, 1822 (Achatinidae). Em ambiente natural e sob condições favoráveis, o período médio de incubação é de 24,42 dias. Os autores encontraram uma relação direta entre o tempo de estivação a que esse molusco foi submetido e o tempo de incubação dos seus ovos. Estivação por 15 dias retardou a oviposição em 1,35 dias em $A$. fulica. Após estivação por 105 e 150 dias, nenhum filhote foi obtido. As reservas energéticas dos moluscos são utilizadas durante o período de estivação para manter uma taxa mínima de metabolismo. Quanto maior o tempo de estivação maior a quantidade de reservas utilizada e conseqüentemente, maior o tempo requerido para o animal compensar este gasto energético e reassumir as atividades biológicas normais (RAUT \& GHOSE 1980).

Os indivíduos da caixa 3 (terra vegetal) apresentaram comprimento de concha menor que os indivíduos das caixas 1 (areia) e 2 (argila) aos 15, 30 e 45 dias de vida (Tab. I).

O teste ANOVA $(p<0,05)$, seguido pelo teste de Tamhane, mostrou haver diferença significativa entre as médias de comprimento de concha dos indivíduos das caixas 1 e 3 aos 15 dias de vida (diferença entre as médias: 1,7356; sig.: 0,000), aos 30 dias de vida (diferença entre as médias: 2,0268; sig.:0,000) e aos 45 dias de vida (diferença entre as médias: 1,1292; sig.: 0,049); assim como entre os indivíduos das caixas 2 e 3 aos 15 dias de vida (diferença entre as médias: 1,6458; sig.: 0,000), aos 30 dias de vida (diferença entre as médias: 1,9958; sig.: 0,000) e aos 45 dias de vida (diferença entre as médias: 1,0958; sig.:0,041). Não houve diferença significativa entre as médias do número de ovos produzidos no primeiro evento reprodutivo dos indivíduos das três caixas (Tab. III).

Estes resultados evidenciam que a capacidade de retenção de água dos substratos não foi o fator de que determinou as diferenças de crescimento entre os indivíduos mantidos nos diferentes substratos. Os indivíduos criados na caixa com areia, que apresenta menor capacidade de retenção de água cresceram mais do que aqueles criados na caixa com terra vegetal, que apresenta boa capacidade de retenção, e tanto quanto aqueles criados na caixa com argila. Essa diferença no crescimento dos moluscos do grupo tratado corrobora os resultados do estudo realizado por D'ÁvILA \& BESSA (2005b), que verificaram que moluscos da espécie $S$. octona criados em terra vegetal cresceram menos do que aqueles criados em areia e argila. As autoras atribuíram esse resultado a um maior consumo de substrato, em detrimento do consumo de ração, pelos moluscos criados em terra vegetal.

\section{CONSIDERAÇÕES FINAIS}

Os resultados do presente estudo evidenciam dois aspectos relacionados ao substrato que influenciam o crescimento e a reprodução de $S$. octona: a capacidade de retenção de umidade e a sua utilização como recurso alimentar.

\section{REFERÊNCIAS BIBLIOGRÁFICAS}

Cook, A. 2001. Behavioral ecology, p. 447-488. In: G.M. BARKER (Ed.). The biology of terrestrial mollusks. New Zeland, CABI Publishing, 558p.

D'Ávila, S. \& E.C. DE A. Bessa. 2005a. Influência do substrato sobre a reprodução de Subulina octona (Brugüière) (Mollusca, Subulinidae), sob condições de laboratório. Revista Brasileira de Zoologia, Curitiba, 22 (1): 197-204.

D'Ávila, S. \& E.C. DE A. Bessa. 2005b. Influência do substrato sobre o crescimento de Subulina octona (Brugüière) (Mollusca, Subulinidae), sob condições de laboratório. Revista Brasileira de Zoologia, Curitiba, 22 (1): 205-211.

Dimitrieva, E.F. 1975. The influense of temperature and moisture of the upper soil layer on the hatching intensity of the slug Deroceras reticulatum Müller. Malacological Review, Ann Arbor, 10: 32-45.

Dundee, S.D.; M. Tizzard \& M. Traub. 1975. Agregative behavior in veronicellide slugs. The Nautilus, Flórida, 89 (93): 69-71.

Elwell, A.S. \& M.J. Ulmer. 1971. Notes on the biology of Anguispira alternata (Stylommatophora: Endodontidae). Malacologia, Ann Arbor, 11 (1): 199-216.

Furtado, M.C.V.; E.C.A. Bessa \& M.C.M. Castañon. 2002. Hystological characterization of ovotestis of Bradybaena similaris (Férussac,1821) (Mollusca, Xanthonychidae) in different developmental phases, kept isolated or in groups, under laboratorial conditions. Revista Brasileira de Zoociências, Juiz de Fora, 4 (2): 229-300. 
Hodasi, J.K.M. 1979. Life story studies of Achatina (Achatina) achatina (Linné). Journal of Molluscan Studies, Londres, 45: 328-339.

HodasI, J.K.M. 1982. The effects of different ligth regimes on the behaviour and biology of Achatina (Achatina) achatina (Linné). Journal of Molluscan Studies, Londres, 48: 283293.

Leahy, W. 1980. Aspectos adaptativos de Bradybaena similaris Ferussac, 1821 (Mollusca, gastropoda, Pulmonata) submetido ao jejum e dessecação. Boletim de Fisiologia Animal, São Paulo, 5: 47-55.

Leahy, W. 1983. Comportamento e características anatomofuncionais da reprodução em Bradybaena similaris (Molusco pulmonado). Ciência e Cultura, São Paulo, 36 (98): 1389 1392.

Pieri, O. \& P. Jurberg. 1981. Aspectos etológicos na sobrevivência dos caramujos vetores da xistosomose ao tratamento com moluscicidas. Memórias do Instituto Oswaldo Cruz, Rio de Janeiro, 76 (1): 47-55.

Raut, S.K. \& K.C. GHose. 1980. Factors influencing gestation lenght in two land snails, Achatina fulica e Macrochlamys indica. Malacological Review, Ann Arbor, 13: 33-36.

Raut, S.K. \& A. Panigrahi. 1988. Egg-nesting in the garden slug Laevicaulis alte (Férussac) (Gastropoda, Soleolifera). Malacological Review, Ann Arbor 21: 101-104.
RichARDOT, M. 1977a. Ecological factors inducing estivation in the freshwater limpet Ferissia waltieri (Basomatophora: Ancylidae).I. Oxygen content, organic matter content and $\mathrm{pH}$ of the water. Malacological Review, Ann Arbor, 10 (12): 159-170.

RichARDOT, M. 1977b. Ecological factors inducing estivation in the freshwater limpet Ferissia waltieri (Basommatophora: Ancylidae). II. Photoperiod, light intensity and water temperature. Malacological Review, Ann Arbor, 10 (1-2): 171-179.

RichARDOT, M. 1978. Ecological factors inducing estivation in the freshwater limpet Ferissia waltieri (Basommatophora: Ancylidae). III. Density levels and food supply. Malacological Review, Ann Arbor, 11 (1-2): 113-119.

Storey, K.B. 2002. Life in slow lane: molecular mechanisms of estivation. Comparative Biochemistry and Physiology. Part A: Molecular and Integrative Physiology, Vancouver, 133: 733-754.

TuAN, R. \& L.C.G. SimÕEs. 1989. Spermatogenesis and desiccation in Biomphalaria tenagophila (Orbigny, 1835) (Gastropoda, Planorbidae). Revista Brasileira de Genética, Ribeirão Preto, 12 (4): 881-885.

WikTor, A. 1987. Spermatophores in Millacidae and their significance classification (Gastropoda, Pulmonata). Malacologische Abhandlungen, Dresden, 12 (9): 1-100.

Recebido em 20.IV.2004; aceito em 11.V.2005. 